$26 \mid 2009$

Varia

\title{
Chasses antiques
}

Jacques Santrot

\section{OpenEdition}

Journals

Édition électronique

URL : http://journals.openedition.org/rao/954

DOI : $10.4000 /$ rao.954

ISBN : 978-2-7535-1609-0

ISSN : 1775-3732

Éditeur

Presses universitaires de Rennes

Édition imprimée

Date de publication : 31 décembre 2009

Pagination : 315-316

ISBN : 978-2-7535-1086-9

ISSN : 0767-709X

\section{Référence électronique}

Jacques Santrot, «Chasses antiques », Revue archéologique de l'Ouest [En ligne], 26 | 2009, mis en ligne le 31 décembre 2011, consulté le 04 décembre 2020. URL : http://journals.openedition.org/rao/954 ; DOI : https://doi.org/10.4000/rao.954 
Giumlia-Mair, A., 2003 - La Necropoli de Misincinis Tavagnacco, Arti Grafiche Friulane SpA, (3 vol.), 150 p.

Cette publication est consacrée à l'étude de l'importante nécropole à incinération de Misincinis, située à proximité du village de Paularo, dans la vallée du Chiarso qui entaille les premiers contreforts des Alpes friulanes au nord-est de la plaine du Pô. Datée de l'âge du Fer, elle présente la précieuse particularité d'avoir été entièrement fouillée et d'avoir suscité de nombreuses études sur son mobilier et son environnement. L'ouvrage présenté ici est constitué de trois volumes, le premier (Dopo lo scavo) étant consacré aux résultats de la campagne de fouille (1995-1997).

Le deuxième (La metallurgia nell'età del ferro) traite de la fabrication et de la composition de certains objets métalliques déposés dans les tombes. Dans un premier temps, après une présentation typologique des fibules, l'auteur présente l'aire de répartition de ces objets en Italie du nord, dans un espace situé entre le nord-ouest de la plaine du Pô, les Alpes orientales et les territoires balkaniques septentrionaux. L'auteur s'interroge alors sur l'origine du métal de ces objets. En répertoriant les mines de cuivre des Alpes, on ne peut que se rendre compte de la proximité de ces sites avec les mines. S'en suit une discussion concernant la nature des minerais et leur mode d'exploitation, ainsi que sur les techniques métallurgiques, en prenant exemple des découvertes faites sur d'autres sites alpins, tel que celui de Mitterberg en Autriche. Enfin, vient l'étude des fibules elles-mêmes, analysées par activation neutronique et par microscopie électronique à balayage. Il en résulte que ce sont des objets composés par un alliage à $\mathrm{Cu} / \mathrm{Sn}$ majoritaires avec une teneur en étain variant de 8 à $12 \%$. Le plomb peut être présent en faible quantité, jusqu’à $3 \%$. Des reconstitutions expérimentales ont été réalisées afin de reproduire le même métal et de retrouver les gestes techniques des bronziers ayant conduit à la fabrication des fibules, de certains pendentifs anthropomorphes ou de chaînettes.

Le troisième volume (L'ambiente e l'uomo nell'età del ferro) est consacré à l'interaction entre l'homme et son environnement. Il s'intéresse aux restes végétaux et humains retrouvés dans les tombes. Ceci a permis de reconstituer le paysage végétal, composé essentiellement de hêtres et de pomoïdés dans un environnement montagnard. L'étude des restes osseux conduit l'auteur à discuter des gestes funéraires mais permet également un recensement des individus par genre et classe d'age ainsi que l'obtention de quelques données concernant d'éventuelles pathologies. Les analyses chimiques de ces mêmes restes permettent également de proposer des hypothèses quant à la nutrition de cette population.

Cécile Le Carlier de VesLud

Jean Trinquier et Christophe Vendries (dir.), 2009 - Chasses antiques. Pratiques et représentations dans le monde gréco-romain (IIt siècle av. IV siècle apr. J.-C.), Actes du colloque international, université de Rennes 2, 20-21 septembre 2007, Rennes, PUR, coll. "Archéologie \& Culture », 276 p. 28 x 22 cm, ill. n. \& b., VIII pl. coul., orientations bibliographiques, indices, résumés (ISBN 978-2-7535-0835-4; $24 €$ ). (publication soutenue par la Fondation de la Maison de la Chasse et de la Nature).

Ce recueil évoque le statut du chasseur et l'art de la chasse dans la Grèce hellénistique et dans le monde romain, avec ses codes et ses rites, son rôle économique et ses aspects juridiques et religieux. Dans leur avant-propos, les responsables du colloque posent d'abord la question du rapport de l'homme avec le monde sauvage, puis s'interrogent sur la place de la chasse dans la hiérarchie des activités sociales à l'époque romaine et sur le dialogue entre nature et culture. Treize contributions sont réparties en trois parties.

La chasse et le pouvoir

Dans une réflexion sur "L'empereur romain et la chasse ", Patrick Le Roux montre que la chasse, habituellement peu prisée des empereurs romains, a compensé, pour Hadrien, l'absence de pratique guerrière. Il rend sa référence militaire à la statue équestre lacunaire de Misène figurant Domitien et remployée avec la tête de Nerva, que S. L. Tuck considérait comme une statue d'empereur chasseur.

Christophe Badel observe " La noblesse romaine et la chasse " : pour l'aristocratie romaine, la chasse est un loisir courant, qui met en œuvre la résistance, la patience et l'endurance, et non une activité ennoblissante, identitaire, faisant appel aux valeurs civiques et à la noblesse de caractère.

François Baratte évoque "La chasse dans l'iconographie des sarcophages, signe social ou valeur funéraire? ». Parmi les quelque 250 sarcophages à scènes de chasse recensés, il est souvent difficile de distinguer les scènes mythologiques des figurations réalistes et des spectacles de venatores, de faire la part entre le puissant symbolisme du triomphe 
sur la mort et l'éventuelle allusion à la passion du défunt pour la chasse. Entre 150 et la fin du Iv ${ }^{e}$ siècle, les scènes de chasse sont souvent en rapport avec la position sociale du défunt et jouent sur l'ambigüité entre mythologie et réalisme, mais affirment presque toujours l'espérance de la victoire sur la mort.

Roger Hanoune traite des "Chasses en Afrique romaine, le cas de Bulla Regia (Tunisie) ", site où les représentations cynégétiques des mosaïques de la maison de la Nouvelle Chasse situent les classiques chasses aux fauves des pavements aristocratiques dans le paysage géographique local.

\section{De l'animal au gibier}

Dans Le gibier dans le monde grec, rôles alimentaire, économique et social, et en s'appuyant tant sur l'archéozoologie que sur la littérature grecque d'époque impériale, Christophe Chandezon évoque le devenir du gibier après la chasse : vendu comme complément de ressources pour les gens de peu, il était couramment quoique modestement consommé par l'ensemble de la population, le grand gibier étant réservé au rituel aristocratique du banquet.

Jean Trinquier aborde « Les chasses serviles, aspects économiques et juridiques » et la question de la commercialisation du gibier, grandement limitée par la durée et le coût du transport. Outre la pratique commune de la chasse par les petites gens de la campagne, ne serait-ce que pour protéger les récoltes, les esclaves ruraux s'adonnaient à la chasse, parfois même sans l'accord de leur maître, mais la capture du gibier était parfois confiée à des esclaves chasseurs, spécialisés en particulier dans la chasse aux oiseaux, qui constituait alors une ressource supplémentaire du domaine, ou qui étaient au service particulier du maitre de famille pour l'accompagner à la chasse ou fournir sa table en gibier.

Dans « L'auceps, les gluaux et l'appeau, à propos de la ruse et de l'habileté du chasseur d'oiseaux », Christophe Vendries montre l'intérêt des Romains pour l'art de l'oiseleur qui, pour être sans risque ni préparer à la guerre, est admiré par les Anciens pour sa grande ingéniosité (usage de la glu, de pièges, de filets et de rapaces auxiliaires), pour la ruse (appeaux, appelants) et pour l'adresse qu'il requiert.

À partir des restes osseux recueillis sur 147 sites, Sébastien Lepetz dresse un panorama de "La chasse dans le nord de la Gaule, étude archéozoologique ", dominé par les vestiges de lièvre, de cerf et de sanglier. L'apport alimentaire de la chasse est très modeste et, sous le Haut Empire à l'inverse du Bas Empire, le gibier est davantage consommé dans les villes qu’à la campagne.

\section{Chasse et culture (textes et images)}

Sébastien Barbara, dans « Les chiens de l'Epyllium Diomedis (v. 8-19), quelques remarques sur la littérature cynégétique à l'époque hellénistique ", traite des chiens de berger, de garde et de chasse d'après la liste des chiens composant la meute de Diomède, où il est question de croisement de races.

Dans « Le motif de la chasse dans les épigrammes de l'Anthologie grecque ", Evelyne Prioux relève, dans les épigrammes dédicatoires (livre VI), nombre d'indications techniques et de termes cynégétiques rares qui démontrent la circulation précoce de traités d'oisellerie. Elle propose que la description des paysages dans les traités cynégétiques ait influencé la grande peinture, tant à l'époque hellénistique que sous le Haut Empire.

Mathilde Simon étudie les apports - modestes - à la connaissance de la chasse "(des) Passages virgiliens relatifs à la chasse et (du) commentaire de Servius ", tandis que Sylvia Estienne, dans Festa Venatica, quels rituels pour la chasse dans le monde romain?, évoque les gestes religieux, les fêtes cynégétiques et les sacrifices aux divinités de la chasse, de Diane en particulier.

Avec « D'un monde à l'autre, la chasse des Pygmées dans l'iconographie impériale ", Véronique Dasen évoque les diverses interprétations - dont le rôle apotropaïque - de ces chasses grotesques dans des paysages nilotiques.

Ces contributions sont complétées, en conclusion, par "Un regard de médiéviste sur les chasses gréco-romaines ", de Baudouin Van Den Abelle, et par l'édition d'un texte rare, la Paraphrase de l'Ixeutique de Denys, précieux traité d'oisellerie copié à Constantinople en 512-513 d'après un manuscrit de la seconde moitié du v viècle, proposée dans une traduction inédite de Bruno Sudan, Pierre Schuwey et Christian Pernet.

Reprenant les études sur les chasses antiques, jusqu'ici principalement fondées sur les sources littéraires et l'iconographie, ce colloque a l'originalité de confronter, quoique timidement, les savoirs classiques aux récents acquis de l'archéozoologie, une discipline prometteuse qui apporte des données quantifiables permettant de relativiser les autres sources, mais qui a elle-même des limites et qui ouvre sur de nouvelles interrogations.

Jacques SANTROT

Conservateur en chef du patrimoine, directeur du musée Dobrée, Nantes 